\title{
La réception du témoignage à l'épreuve du silence. L'aîné des orphelins de Tierno Monénembo
}

Et puis

mieux vaut ne pas y croire

à ces histoires

de revenants

plus jamais vous ne dormirez

si jamais vous les croyez

ces spectres revenants

ces revenants

qui reviennent

sans pouvoir même

expliquer comment.

Charlotte Delbo, Une connaissance inutile (1970)

Le silence et l'inaction de la communauté internationale face au génocide des Tutsis et des Hutus modérés du Rwanda en 1994 est une question qui a de quoi troubler. Devant l'absence de représentation, qui perpétuait ce silence, dans l'imaginaire est née l'initiative « Rwanda : écrire par devoir de mémoire». Parrainée par le festival littéraire Fest'Africa et la Fondation de France, l'opération consistait à permettre à dix écrivains africains d'effectuer un séjour au Rwanda, entre 1998 et 2000, afin de produire des textes sur le génocide. Les auteurs avaient la liberté de la forme et celle, essentielle, de prendre la parole ou de se taire après leur séjour rwandais. Romans, pièces de théâtre, essais et récits ont résulté de cette initiative ${ }^{1}$. Bien qu'elles brisent

Caroline Giguère - étudiante au doctorat en études littéraires à l'Université du Québec à Montréal. Adresse de correspondance : giguere.caroline.3@courrier.uqam.ca.

1. Boubacar Boris Diop, Murambi, le livre des ossements, Stock, 2000; Nocky Djedanoum, Nyamirambo!, Le Figuier Fest’Africa, 2000 ; Monique Ilboudo, Murekatete, Le figuier Fest’Africa, 2000 ; Koulsy Lamko, La phalène des collines, Le serpent à plumes, 2001 ; Tierno Monénembo, L'aîné des orphelins, Seuil, 2000 ; Jean-Marie Vianney Rurangwa, Le génocide des Tutsi expliqué à un étranger, Le Figuier, 2000 ; Véronique Tadjo, L'ombre d'Imana. Voyage jusqu'au bout du Rwanda, Actes Sud, 2000 ; Abdourahman Ali Wabéri, Moisson de crânes. Textes pour le Rwanda, Le serpent à plumes, 2000. 
le silence, ces œuvres ont en commun d'être marquées par les difficultés éthiques et esthétiques de rendre témoignage dans l'après-coup et qui plus est, de l'extérieur ${ }^{2}$. Le roman de Tierno Monénembo, L'aîné des orphelins, relève ses défis en préservant l'opacité de l'expérience du survivant. Ce faisant, il confronte son lecteur à l'indicible et l'invite à s'interroger sur les limites éthiques du devoir de mémoire.

\section{Devoir de mémoire et témoignage "à relais "}

La littérature de la Shoah a ceci de particulier qu'elle n'a pas de précédent, qu'elle présente un défi inédit dans la négociation entre la nécessité morale de témoigner et la difficulté éthique et esthétique de représenter l'horreur. Lorsque le projet «Écrire par devoir de mémoire» est mis sur pieds autour du génocide rwandais, les auteurs et les critiques renvoient à ces récits de survivants des camps qui se présentent alors comme références en matière d'écriture de l'horreur ${ }^{3}$. Cette référence est parfois explicite, comme c'est le cas dans L'aîné des orphelins de Tierno Monénembo ${ }^{4}$, mais le plus souvent, c'est à travers un métadiscours sur la mémoire, l'indicible et le témoignage que la littérature de la Shoah s’inscrit dans le répertoire (Iser, 1985 : 99-127) des textes sur le Rwanda. Le mandat de produire des œuvres par « devoir de mémoire ", auquel souscrivent les écrivains africains participant au projet, tire ses origines de l'engagement à témoigner, pris par les survivants à la libération des camps. Cet engagement a une double visée : rétrospective en ce qu'il repose sur la promesse de rendre hommage aux morts en leur donnant une voix ; et prospective, puisqu'il vise à prévenir la répétition du génocide (Lalieu, $2001: 85)^{5}$. L'engagement à témoigner de l'expérience génocidaire prend une dimension particulière dans le cas des récits publiés du projet "Rwanda : écrire par devoir de mémoire ${ }^{6}$ en raison de la distance des écrivains par rapport aux massacres : ils n'ont pas, eux-mêmes, vécu les événements et leur séjour sur les lieux du génocide, en 1998, est relativement tardif. Ils ont certes rencontré des survivants et visité les lieux commémoratifs, mais comme le souligne Romuald Fonkua, les récits résultant de ce projet constituent

2. Deux dramaturges rwandais faisaient initialement partie du projet Fest'africa : Tharcisse Kalissa Rugano et Jean-Marie Vianney Kayishema. Kayishema n’était pas sur les lieux au moment du génocide et Rugano s'est retiré du projet.

3. Il apparaît important ici de souligner que les auteurs ne prétendent pas penser le génocide rwandais dans les termes de la Shoah, mais s'intéressent plutôt aux défis de la représentation en contexte génocidaire.

4. «- Vous pensez peut-être que tous les rescapés d'Auschwitz se souviennent de la vie qu'ils ont menée avant de goûter à l'enfer ? » (2000: 107).

5. Cet article de Lalieu reconstitue la trajectoire du « devoir de mémoire » depuis ses origines jusqu'aux récupérations actuelles.

6. Pour une mise en perspective historique sur la notion d'engagement et sur le témoignage en littérature africaine, voir : Jean-Pierre Karegeye, « Rwanda : Littérature post-génocide, écritures itinérantes : témoignage ou engagement ? », Protée, 37 (2), 2009, p. 21-32. 
des « témoignages du dehors " (Brézault, $2003: 5$ ) et se distinguent des récits oraux ou écrits des survivants ${ }^{7}$. L'exercice de devoir de mémoire, auquel les écrivains se livrent, est donc de "deuxième main » : ayant constaté les preuves matérielles et ayant reçu les témoignages de survivants, ils s'engagent à transmettre au monde leur expérience. Bien qu'ils n'aient pas vécu le génocide, leur posture se rapproche de celle des témoins mémorialistes, éloignés dans le temps (et ici dans l'espace), qu'évoque Alain Goldschläger : leur distance leur permet une posture réflexive ; ils se donnent comme mission de parler au nom d'une collectivité ; et ils offrent une "lecture dirigée des événements » (2001 : 267-269). Ces écrivains sont en ce sens d’abord des lecteurs ou du moins des récepteurs qui, pour reprendre les termes de Goldschläger, assument le fait d'être " dépositaire[s] d'un héritage » : " le lecteur [de la littérature de témoignage de la Shoah] se doit d'assumer la charge d'héritier et à ce titre s'engager à propager et à transmettre l'héritage, se transformant dès lors en chaînon d'un système de communication et de mémoire " $(2001: 265)$. Le caractère dialogal du témoignage, sur lequel insiste Paul Ricoeur (2000 : 205), est ainsi inscrit d'emblée dans la démarche d'écriture qui constitue un acte de réception et "d'accréditation " des récits de survivants. Ces récits trouvent ainsi leur existence et leur légitimité, parce qu'ils ont été entendus et qu'ils seront " relayés " par les écrivains dans une chaîne de transmission mémorielle. Dans ce contexte, les œuvres écrites dans le cadre du projet de Fest'Africa assignent une place particulière à leurs lecteurs envisagés comme dépositaires d'une mémoire dont la finalité dépasse les considérations esthétiques et s'inscrit dans un engagement éthique.

\section{Témoigner : la double contrainte de l'engagement et de l'indicible}

Or, l'établissement d'une chaîne testimoniale ne va pas de soi. Les critiques (Bazié, 2004 ; Semujanga, 2003, 2008) n'ont pas manqué de souligner l'horizon d'attente créé par les récits concentrationnaires en revenant, entre autres, sur le dilemme entre le caractère indicible de l'expérience concentrationnaire et la nécessité morale de prendre la parole, justement au nom du devoir de mémoire ${ }^{8}$. Sans prétendre résumer ce débat complexe, il est nécessaire pour notre propos de retenir donc que certains auteurs prennent le parti pris de la clarté $\left(\mathrm{Levi}^{9}\right)$ ou de la fiction $\left(\right.$ Semprun $\left.^{10}\right)$ pour représen-

7. La pièce Rwanda 94 de Groupov, s'ouvrant sur le témoignage de Yolande Mukagasana, constituerait l'exemple extrême d'un « témoignage du dedans ». Mukagasana a aussi publié deux récits autobiographiques : La mort ne veut pas de moi (1997) et N'aie pas peur de savoir (1999).

8. Pour une réflexion plus large sur ce dilemme, voir entre autres : Dans la langue de personne (Ertel, 1993) et Les récits du génocide: Sémiotique de l’indicible (Rinn, $1998: 19-35)$.

9. Si c'est un homme, Paris, Juliard, 1987 et Les naufragés et les rescapés, Paris, Gallimard, 1986.

10. L’écriture ou la vie, Paris, Gallimard, 1994. 
ter leur vécu des camps; d'autres auteurs soutiennent que la Shoah échappe à la représentation $\left(\right.$ Adorno $^{11}$, Lanzmann ${ }^{12}$ ). Cet interdit de représentation serait à la fois éthique, puisque figurer l'horreur du génocide le banaliserait, et esthétique, car les ressources langagières ne suffiraient pas pour exprimer la Shoah. Certains auteurs du projet «Écrire par devoir de mémoire » reprennent les termes de ce débat, à l'instar d'Abdourahman Waberi qui paraphrase Paul Celan en se demandant, en préface Moisson de crânes, " comment écrire après le génocide rwandais ? " et en s'interrogeant du même souffle : "On se dit que la littérature, cette fabrique d'illusions, avec ses suspensions d'incrédulité, reste bien dérisoire. On se dit que peut la fiction dans une telle situation" $(2000: 16)$. Boubacar Boris Diop publie, quant à lui, un chapitre, intitulé "Génocide et devoir d'imaginaire ", dans son essai L'Afrique au-delà du miroir (2007: 17-35). Si chacun des auteurs du projet négocie à sa manière cette tension entre le dire et le taire (Rinn, 1998 : 19-26), le roman L'aîné des orphelins de Tierno Monénembo garde inscrit en son centre cette dynamique et entretient un certain silence autour du génocide lui-même, plaçant son lecteur face aux trous de mémoire et aux mensonges d'un narrateur dont le témoignage pourrait être considéré comme « défaillant» (Mazauric, 2007 : 15).

Pourtant, dès ses seuils (Genette, 1987), le roman de Monénembo semble prendre le parti de dire et de s’engager à travers la fiction : «Si le génocide rwandais est irréfutable, les situations et les personnages de ce roman sont, eux, fictifs pour la plupart » (2000 : exergue, nous soulignons). Le recours à l'imaginaire pour rendre compte de l'horreur sénonce ici circonscrit de précautions respectueuses de la nécessité d'ancrages référentiels portant la dimension éthique du projet deécriture. Le récit répond à cet équilibre entre réalisme et libertés romanesques : le personnage principal fictif, Faustin, traverse le territoire rwandais, évoqué en une toponymie précise, et les dates du génocide sont marquées, bien que presque toujours sans la mention du mois d'avril (2000 : $45,75,142,143,145)$. De même, le péritexte éditorial, auquel Gérard Genette attribue la fonction de promouvoir le texte et d'en orienter la lecture (1987: 18-36 et 111-124), conforte l'horizon d'attente réaliste et engagé du témoignage, aussi fictif et relayé soit-il. D’abord, la photographie de Raymond Depardon, choisie pour lédition de la collection " Points ", représente non seulement un enfant survivant du génocide rwandais, mais plus encore du massacre de Nyamata ${ }^{13}$. Il faut ajouter à ceci une prière d'insérer en quatrième de couverture qui révèle d'emblée le récit du génocide que l'écriture romanesque travaille à retarder et à ne rendre que de manière diffractée :

11. «Critique de la culture et société » in Prismes, Paris, Payot, 2003.

12. "Holocauste, la représentation impossible ", Le monde, 3 mars 1994.

13. L'enfant en question est Casius Niyonsaba, douze ans, dont le témoignage est rapporté par Jean Hatzfeld dans son recueil intitulé Dans le nu de la vie : Récits des marais rwandais (2000 : 15-21). La photographie qui accompagne son témoignage est très semblable à celle qui a été retenue pour faire la couverture de L'aîné des orphelins, à la différence près qu'on y voit le profil de l'enfant. Le choix d'un portrait de dos pour le roman donne certes un caractère anonyme à la photo, mais il n'en reste pas moins que le drame du jeune Niyonsaba est au plus près de celui du personnage principal de Monénembo. 
Au Rwanda, Faustin Nsenghimana, né d'un père hutu et d'une mère tutsie, est l'aîné de quatre enfants. [...] Il a treize ans lorsque des hommes entourent les collines de Nyamata et exhortent les gens à aiguiser les machettes et les couteaux. Ses parents sont massacrés, il prend la fuite, mène une vie errante et misérable pendant des mois. Et lorsqu'enfin il retrouve ses frères et sœurs, sa vie est de nouveau bouleversée... (Monénembo, 2000)

Tout y est livré de l'identité et du drame de Faustin : sa double appartenance « ethnique », le funeste destin de ses parents, ainsi que ses retrouvailles avec sa fratrie. Comme si cela ne suffisait pas, la prière d'insérer de la première édition du roman est reproduite en page de garde dans l'édition de la collection « Points». Bien que plus fidèle à la structure romanesque que nous avons évoquée, reproduisant entre guillemets un texte attribuable à l'auteur, l'insistance est mise sur le fait que Faustin soit en attente de son exécution: «Moi, Faustin Nsenghimana, je n'en ai plus pour longtemps. Ils viendront me tuer demain ou après-demain [...] Eh oui, j'ai continué à jouer au cerf-volant moi, même quand ils ont entouré les collines et qu'ils ont exhorté les gens à aiguiser les machettes et les couteaux » (Monénembo, 2000). Cette introduction avait encore le mérite de laisser en suspens l'identité des bourreaux et de défier les attentes du lecteur face au récit du génocide qu'il s'apprête à lire : le personnage, qui se présente comme dans un témoignage légal - " Moi, Faustin Nsenghimana... » - pourrait craindre l'arrivée des " génocideurs » comme il pourrait lui-même être des bourreaux et attendre la sanction de ses actes. Toutefois, le propos éditorial ne tarde pas à lever l'indétermination : «Il [Faustin] est emprisonné pour meurtre et attend son exécution. L'adolescent a perdu son innocence depuis longtemps, depuis le jour où ses parents furent massacrés [...] » (Monénembo, 2000). Toutes ces précautions contribuent à consolider l'horizon d'attente face au témoignage du génocide : réalisme, récit à la première personne d'une victime, allusion à la machette - devenue topos des massacres de 1994.

Toutefois, si l'on poursuit la lecture au-delà de ces seuils, les attentes créées sont vite déstabilisées par un récit qui ne se focalise pas sur le génocide, et qui plus est, est produit par un narrateur homodiégétique qui ne souscrit pas au portrait du témoin motivé par le devoir de mémoire. Plusieurs critiques ont évoqué le flou qu'entretient la narration sur le récit du génocide à proprement parlé. Josias Semujanga souligne ainsi une «écriture oblique de l'événement » $(2003: 105)$ qui aurait pour effet de «simuler le silence et $[\mathrm{de}]$ donne[r] au récit sa puissance suggestive " (2003:112). Catherine Mazauric, quant à elle, insiste sur les mensonges et les trous de mémoire de Faustin, " manquements » au témoignage dont le lecteur deviendrait le dépositaire, si ce n'est le juge (2007 : 348). Il est en fait significatif que le narrateur adolescent ne parle pas volontiers du génocide. Dès l'incipit, il avertit : «Quand je pense à cette époque-là, c'est toujours malgré moi » (2000: 15). Faustin névoque cet épisode de sa vie que sous pressions extérieures : lorsqu'il est capturé par le FPR (2000 : 46), dans le délire qui suit ses retrouvailles avec sa fratrie (2000:70-71) et pour répondre aux journalistes qui lui demandent de témoigner devant la caméra (2000 : 105-110). Cette dernière instance, 
la seule dans laquelle le mensonge est sans équivoque, mérite qu’on s'y attarde, parce qu'elle se présente comme une mise en abîme du témoignage et participe à désambiguïser (Dallenbach, 1980) un récit qui multiplie par ailleurs les lieux d'indétermination (Iser, 1985). Faustin y répond à la demande de son « ami » Rodney, le journaliste, en produisant de faux témoignages. Complice du journaliste sans scrupule, Faustin se moque de l'authenticité qu'on accorde à la parole de l'enfant, perçu comme victime parmi les victimes : "Je suis sûr que son témoignage sera meilleur que celui de tous ces adultes qui ne manqueront pas de nous bassiner avec le pourquoi et le comment de tout ce qui est arrivé. Il a vécu les choses, lui, et avec les yeux d'un enfant. La vérité sort de la bouche de l'enfant» (Monénembo, 2000 : 107). Le jeune Faustin n’est pas dupe des injonctions à la parole dont il est l'objet et de l'instrumentalisation éventuelle de ses souffrances par les médias. Il en joue, tout en gardant le silence sur son expérience personnelle. L'adolescent est ainsi trop lucide des enjeux entourant sa parole et il répond à la demande en la détournant. Le jeune Faustin brise ainsi le pacte qui, avant toute production de témoignage, sous-tend la fiabilité de celui-ci, « celle de tenir sa promesse, de tenir parole » (Ricoeur, $2000: 206)$. Ce narrateur, « indigne de confiance » (Mazauric, 2007 : 354), renvoie le lecteur à ses attentes plutôt que d'y répondre : le roman de Monénembo déjoue l'horizon de son public d'une manière semblable à celle dont Faustin joue de celles des journalistes. Plutôt que de présenter la violence directe du génocide, Monénembo choisit de confronter son lecteur à un récit repoussant dans ses limites la « vérité » du drame personnel de son personnage principal et de s'attarder plutôt sur la violence structurelle (Galtung, 1990) qui succède aux massacres et qui dérègle les rapports sociaux de manière durable.

\section{Le silence : une invitation à lire autrement}

Au narrateur « indigne de confiance » que serait Faustin, L'aîné des orphelins fait correspondre un narrataire, un lecteur implicite du même acabit. Bien qu'un effet de familiarité se dégage de la narration qui relance constamment son auditoire en multipliant les questions rhétoriques à son endroit, force est de constater que la proximité apparente de cet auditoire contraste avec son assignation à une position d'extériorité. Cette extériorité se donne à lire d'abord à travers un métadiscours qui se développe dans le texte par la présence d'italiques, lorsque le kinyarwanda est utilisé, italiques souvent suivis d'une explication sommaire du narrateur (Monénembo, $2000: 76,81$, 90, 92). Ce métadiscours donne à penser que le témoignage de Faustin n'est adressé ni à ses compatriotes ni aux juges de son procès, comme aurait pu le laisser penser la prière d'insérer en forme de témoignage judiciaire de la première édition, mais qu'il est plutôt destiné à un lecteur étranger au drame rwandais. Plus encore, la narration sort à quelques reprises de son récit principalement rétrospectif pour interpeller un lecteur défini par son incrédulité et auquel il faut demander : «Croyez-moi [...] » (Monénembo, $2000: 91$ ). Le narrateur rabroue par ailleurs le lecteur avide 
de détails attestant la véracité du récit : "Ne me demandez pas combien de mois s'étaient écoulés! » (Monénembo, 2000 : 47). De la même façon, il repousse du revers de la main les interrogations que pourrait avoir son lecteur face aux motivations derrière son autosabotage : "Quelqu'un me demanderait aujourd'hui pourquoi j’ai fui la Cité des Anges bleus, je ne saurais quoi lui répondre ( (Monénembo, 2000 : 78). Le lecteur implicite de L'aîné des orphelins serait ainsi sceptique face aux demandes d'accréditation du témoin et il (se) poserait des questions sur le pourquoi et le comment d'événements sur lesquels le narrateur n'a pas prise. En cela, ses attentes se rapprochent de celles des journalistes décrits plus haut.

Aussi, contrairement à ce que plusieurs critiques prétendent, ce n'est peut-être pas tant l'amnésie (Semujanga, 2003 : 105) ou le caractère rebelle de Faustin (Mazauric, 2007 :352353) qui expliquent le silence que le récit entretient sur l'expérience du génocide, puisque au tiers à peine du roman, l'adolescent se livre à une longue " confession » :

Je fis un effort surhumain pour revenir sur les fameux avènements que ma mémoire ne voulait plus revoir. Soudain, tout séclaircit. Ma bouche s'ouvrit toute seule et je parlai si vite qu'il [le capitaine] m'arrêta pour faire venir mon vieux compagnon de route.

- [...] Prends un magnétophone et enregistre-moi tout ce que dira ce jeune homme (Monénembo, $2000:$ 46).

Non seulement le témoignage de Faustin trouve-t-il des auditeurs qui lui accordent crédit, mais le récit nous apprend que sa « confession » est archivée par le FPR. Or, le récit romanesque fait ellipse et la longue confession de Faustin ne sera jamais livrée au lecteur, placé comme dans la position d'un tiers extérieur à la relation testimoniale qui s'installe entre Faustin et les représentants du FPR. Le lecteur de L'aîné des orphelins est, en fait, confronté à plusieurs lieux d'indétermination prenant la forme d'ellipses qui rendent partielle sa saisie de la trajectoire de Faustin ${ }^{14}$. Tout au long du roman, le génocide lui-même n’est désigné que de manière métonymique par l'expression : « quand le brigadier Nyumurowo s'empara de mon cerf-volant » (Monénembo, $2000:$ 75, 76, 92, 117, 141, 155). Illustrant à la fois la fin de l'innocence et le début des violences génocidaires pour la famille de Faustin, ce n'est qu'en conclusion du roman que l'évocation du cerf-volant confisqué est réinscrite et trouve sa signification dans le déroulement global du génocide de Nyamata ${ }^{15}$. Tout

14. L'arrivée de Faustin à Kigali et les raisons pour lesquelles il se sépare du FPR auprès duquel il prétendait pourtant avoir "trouvé une nouvelle famille " (2000:46) restent nébuleuses. Au-delà du destin de Faustin, le lecteur ne saura jamais comment ses sœurs et son frère ont survécu au massacre de Nyamata, ni ce qui leur arrive après l'emprisonnement de leur aîné.

15. Il est aussi significatif que le récit du massacre de l'église de Nyamata, auquel a survécu Faustin, ne se fait que par l'entremise du recours à la mémoire collective, l'anamnèse individuelle du personnage principal n'advenant finalement jamais : « Mes souvenirs du génocide s'arrêtent là. Le reste, on me l'a raconté par la suite ou alors cela a rejailli tout seul dans ma mémoire en lambeaux, par à-coups, comme des jets d'eau boueuse jaillissant d'une pompe obstruée » (Monénembo, $2000: 156$, nous soulignons). 
en les laissant constamment sous-entendre, l'écriture romanesque garde sciemment les violences génocidaires hors-champ, procédant à l'inverse du sensationnalisme médiatique mis en évidence dans la mise en abîme des faux-témoignages de Faustin. Le lecteur est ainsi amené à remettre en question les attentes éveillées non seulement par le genre testimonial, mais aussi renforcées par le péritexte éditorial et plus largement par le projet "écrire par devoir de mémoire ». Certes, on pourrait penser que le fait de n'en venir au génocide lui-même qu'à la toute fin du roman entretient une forme de suspense, mais le récit lacunaire, dont nous venons de faire état, invite davantage à penser que Monénembo confronte ainsi son lecteur à la nature du témoignage lui-même, " partiel, subjectif et forcément incapable de dire l'événement dans sa totalité et son authenticité [...]. » (Goldshläger, 2001 : 275). Goldshläger ajoute que ceci «n'est guère pour tenter quelqu'un qui désire connaître l'événement » (2001 : 275, nous soulignons). Or, et c'est la force du récit de Monénembo, L'aîné des orphelins demande à son lecteur de "faire avec " les silences de Faustin. Il exige du lecteur de composer avec un passé narratif qui ne se livre pas tout entier, qui ne comble pas ses zones d'indétermination. Dans cette négociation " entre ce qui est dit et ce qui se donne à entendre " (Iser, $1985: 87$ ), le récit produit son effet le plus marquant : il donne à expérimenter la perte, le manque. Les « blancs » ainsi préservés ne sont pas des déficiences ou des maladresses du témoignage, ils sont ce par quoi s'établit la chaîne de transmission, puisque "l'indétermination se présente comme une condition de communication qui produit une interaction au cours de laquelle le texte peut être vécu » (Iser, 1985 : 55). La question que pose le texte de Monénembo est celle de la transmission de l'indicible : par-delà les faits avérés des massacres, comment recevoir les silences du survivant et comment s'en faire le digne héritier (Goldshläger, $2001: 215)$ ?

En décevant les attentes d'un lecteur en quête d'une certaine connaissance, L'âiné des orphelins propose une autre réception du génocide qui considérerait l'onde de choc dans une temporalité qui excède les massacres et prendrait en compte, par exemple, la violence structurelle d'un système pénitencier qui condamne un enfant (de surcroît, un enfant rescapé d'un génocide) à mort. En revenant aux seuils du roman, on constate que si Monénembo s'engage dans une entreprise de devoir de mémoire, il donne présence à la visée prospective de ce travail, en dédiant son roman à « tous les Rwandais, Twas, Hutus ou Tutsis... et vivants de préférence " (dédicace, nous soulignons). Ce choix d'écrire pour les vivants inscrit l'œuvre dans l'actualité post-génocidaire et oriente ses enjeux vers une reconstruction dont la trajectoire de Faustin montre la précarité. 


\section{BIBLIOGRAPHIE}

Bazié I. 2004. Au seuil du chaos : devoir de mémoire, indicible et piège du devoir dire. Présence francophone 63. 29-45.

Brezault É. 2003. Raconter l'irracontable : le génocide rwandais, un engagement personnel entre fiction et écriture journalistique. Éthiopiques : Revue négro-africaine de littérature et de philosophie 71. 1-8.

Dällenbach L. 1980. Réflexivité et lecture. Revue des sciences humaines 177. 23-37.

Davis C. 1999. Littérature de l'holocauste et éthique de la lecture. Études littéraires 31(3). 57 68.

Diop B. B. 2006. L'Afrique au-delà du miroir. Paris. Philippe Rey.

Galtung J. 1990. Cultural Violence. Journal of Peace Research 27(3). 291-305.

Genette G. 1987. Seuils. Paris. Seuil.

Goldschläger A. 2001. La littérature de témoignage de la Shoah : Dire l'indicible - Lire l'incompréhensible. Texte 19-20. 259-278.

Hatzfeld J. 2000. Dans le nu de la vie : récits des marais rwandais. Paris. Seuil.

Iser W. 1985. L'acte de lecture : théorie de l'effet esthétique. Bruxelles. Mardaga.

Lalieu O. 2001. L'invention du « Devoir de mémoire». Vingtième Siècle. Revue d'histoire 69. 83-94.

Mazauric C. 2007. Les mensonges de la mémoire : la part du lecteur dans Le Cavalier et son ombre de Boubacar Boris Diop et L'Aîné des orphelins de Tierno Monénembo. In P. Halen \& J. Walter (dir.). Les langages de la mémoire: Littérature, médias et génocide au Rwanda. Metz. Université Paul Verlaine-Metz Centre de recherches «Écritures ». 341-356.

Ricoeur P. 2000. La mémoire, l'histoire, l’oubli, Paris. Seuil.

Rinn M. 1998. Les récits du génocide: Sémiotique de l'indicible. Lausanne. Delachaux et Niestlé. Semujanga J. 2003. Les méandres du récit du génocide dans L'aîné des orphelins. Études littéraires 35(1). 101-115.

Waberi A. 2000. Moisson de crânes: Textes pour le Rwanda. Paris. Le serpent à plumes.

\section{The testimonial reception to the test of silence}

ABSTRACT: From the perspective of the reader-response criticism, this article underlines the ethical and esthetical tension between the duty of memory and the unspeakable in the context of the project "Rwanda : écrire par devoir de mémoire » and specificly in the novel L'aîné des orphelins by Tierno Monénembo. Although the " horizon » of expectations typical to the commitment toward the duty of memory is reinforced by the peritext (Genette, 1987), Monembo's novel challenges the reader's expectations by silencing and delaying the personnal story of young narrator-survivor Faustin. This stepback from direct violence allows the novel to focus on structual forms of violence (Galtung 2010) and urges the public to question his responsability in the testimonial reception.

Keywords: silence, genocide, Rwanda, testimony, reader-response criticism. 